\title{
High harmonic generation in the relativistic limit
}

\author{
B. DROMEY ${ }^{1}$, M. ZEPF ${ }^{1 *}$, A. GOPAL ${ }^{2}$, K. LANCASTER ${ }^{2}$, M. S. WEI ${ }^{2}$, K. KRUSHELNICK ${ }^{2}$, M. TATARAKIS ${ }^{3}$, \\ N. VAKAKIS ${ }^{4}$, S. MOUSTAIZIS ${ }^{4}$, R. KODAMA ${ }^{5}$, M. TAMPO ${ }^{5}$, C. STOECKL ${ }^{6}$, R. CLARKE ${ }^{7}$, H. HABARA ${ }^{7}$, \\ D. NEELY7 , S. KARSCH7 AND P. NORREYS ${ }^{7}$
}

\author{
${ }^{1}$ Department of Physics and Astronomy, Queen's University Belfast BT7 1NN, UK \\ ${ }^{2}$ Blackett Laboratory, Imperial College, London SW7 2BZ, UK \\ ${ }^{3}$ Department of Electronics, Technological Educational Institute of Crete, 73133 Chania, Greece \\ ${ }^{4}$ Technical University of Crete, Institute of Matter Struct. \& Laser Phys. Chania, 73132 Chania, Greece \\ ${ }^{5}$ ILE University of Osaka, 565-0871 Osaka, Japan \\ ${ }^{6}$ Laboratory for Laser Energetics, University of Rochester, Rochester, New York 14623-1299, USA \\ ${ }^{7}$ Central Laser Facility, CCLRC Rutherford Appleton Laboratory, Chilton, Didcot OX11 0QX, UK \\ *e-mail:m.zepf@qub.ac.uk
}

5 he generation of extremely bright coherent $\mathrm{X}$-ray pulses in the femtosecond and attosecond regime is currently one of the most exciting frontiers of physics-allowing, for the first time, measurements with unprecedented temporal resolution ${ }^{1-6}$. Harmonics from laser-solid target interactions have been identified as a means of achieving fields as high as the Schwinger $\operatorname{limit}^{2,7}\left(E=1.3 \times 10^{16} \mathrm{~V} \mathrm{~m}^{-1}\right)$ and as a highly promising route to high-efficiency attosecond $\left(10^{-18} \mathrm{~s}\right)$ pulses $^{8}$ owing to their intrinsically phase-locked nature. The key steps to attain these goals are achieving high conversion efficiencies and a slow decay of harmonic efficiency to high orders by driving harmonic production to the relativistic limit ${ }^{1}$. Here we present the first experimental demonstration of high harmonic generation in the relativistic limit, obtained on the Vulcan Petawatt laser'. High conversion efficiencies $\left(\eta>10^{-6}\right.$ per harmonic) and bright emission ( $>10^{22}$ photons $\mathrm{s}^{-1} \mathrm{~mm}^{-2} \operatorname{mrad}^{-2}(0.1 \%$ bandwidth $)$ ) are observed at wavelengths $<4 \mathrm{~nm}$ (the 'water-window' region of particular interest for bio-microscopy).

In the case of attosecond pulse generation, the conversion efficiency into a given pulse is, of course, much higher than for individual harmonics (between 10-100 times), because the spectral width required to form such a pulse spans many harmonic orders $^{8}$. The observed spectra also contain evidence of $>850$ th order of $1,054 \mathrm{~nm}$ fundamental $(\sim 1.2 \mathrm{~nm},>1 \mathrm{keV})$, highlighting the potential of this source for applications requiring high peak brightness in the soft X-ray region. This high promise has led to harmonics from solid targets forming an integral part of current proposals for a European attosecond light source ${ }^{10}$.

The nonlinear medium underlying the harmonic generation process is the plasma-vacuum interface that forms at the surface of the solid target. The harmonics are generated by the motion of the electrons in the laser field at the plasma-vacuum interface ${ }^{11,12}$. Qualitative insight can be gained by considering the interface between the plasma critical-density surface and the vacuum as an oscillating mirror ${ }^{12,13}$, whereby the mirror oscillation is driven by the laser field acting on the electrons that form the mirror. The two main driving terms are the electric field of the laser (at the laser frequency $\omega$ ) and the ponderomotive force or light pressure (at $2 \omega$ ), leading to both odd and even harmonics being produced. From this model most of the key features of the harmonic generation process can be readily understood.

First, the oscillating mirror modulates the phase of the reflected light wave (because the reflection point varies with time across the optical cycle). The reflected electromagnetic waveform is now no longer sinusoidal and consequently contains many highorder harmonics. The conversion efficiency of each harmonic can be calculated from the Fourier transform of the reflected waveform and has previously been observed to scale as $\eta(n) \sim$ $n^{-p}$ (where $n$ is the harmonic order; previous observations ${ }^{14}$ have observed values of $p=5.5 \ldots 3.3$ for an irradiance of $I \lambda^{2}=$ $\left.5 \times 10^{17}-10^{19} \mathrm{~W} \mathrm{~cm}^{-2} \mu \mathrm{m}^{2}\right)$. From these considerations, it is clear that the conversion efficiency into a given harmonic will depend on the amplitude and shape of the mirror excursions and will saturate as the mirror velocity approaches the speed of light (the relativistic limit). Recently, a relativistic limit of $p_{\mathrm{REL}}=2.5-3$ with $p=2.5$ for quasi-monochromatic pulses and $p=3$ for broadband pulses respectively has been predicted ${ }^{2}$, suggesting that substantial improvements in efficiency are possible particularly for the highest orders.

Second, the process can only be efficient if the reflection point is well defined, that is, a step-like plasma-density gradient at the critical-density surface ${ }^{12,15}$. At high intensities such a sharp 


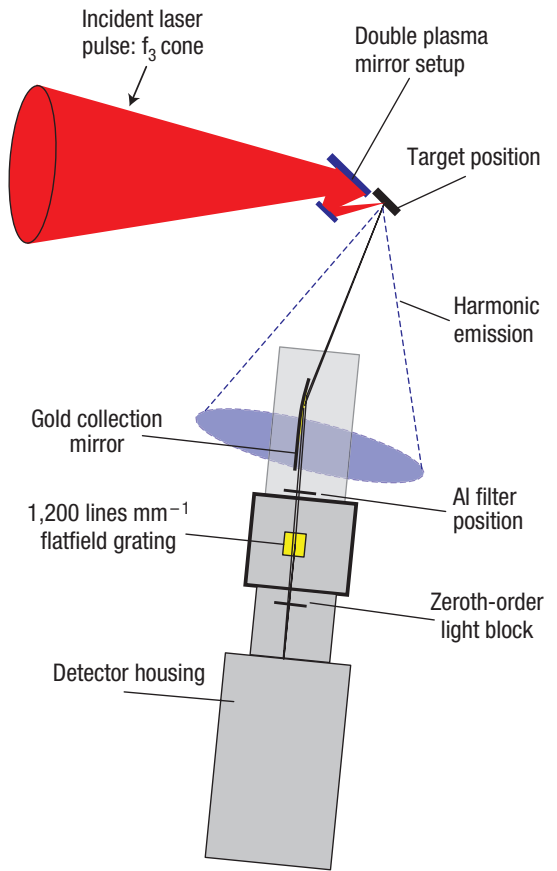

Figure 1 Schematic diagram of the experimental setup. The 600-fs laser pulses were focused using an $\mathrm{f} / 3$ off-axis parabolic mirror, and delivered peak intensities $>10^{20} \mathrm{~W} \mathrm{~cm}^{-2}$ onto the target. The reflected harmonic emission is detected using an extreme-ultraviolet spectrometer consisting of a calibrated, cylindrical 1,200 lines $\mathrm{mm}^{-1}$ Hitachi flatfield grating imaging the source in the spectral dimension, and a cylindrical mirror to image the source in the transverse dimension. A 0.2- $\mu$ m aluminium filter was used to block optical emission and a block was inserted after the grating to prevent scattered zero-order diffraction light from reducing the quality of the observed spectra. The PMs could either be inserted or removed to vary the pulse contrast conditions. Using a double PM configuration enhances the intrinsic pulse contrast by $>10^{4}$ and results in a reduced pulse rise time $^{18,19}$. Approximately $40 \%$ of the laser energy incident of the first PM is incident on the target.

boundary can be achieved in two distinct ways. Either the pulse has a sufficiently rapid rise time to keep the initial solid-vacuum interface intact, or the pulse is of sufficient intensity and duration to steepen the shallow plasma gradient that has been produced by the prepulse (energy reaching the target before the desired pulse). The latter regime has only been observed ${ }^{14-16}$ using pulse durations of $>\sim 1$ ps and is not applicable here.

Third, the cycle-averaged shape of the mirror surface determines the angular distribution of the reflected light. For the harmonics to be reflected into the specular direction the mirror needs to be sufficiently flat. The initial surface roughness can easily be made small enough to fulfil this condition. However, at high intensities the radiation pressure $(\sim 2 I / c$, where $c$ is the speed of light) exceeds the plasma pressure. Depending on the duration and the intensity of the pulse this initially leads to surface denting and subsequently to strong modulations ${ }^{16}$ that will result in the harmonic light being emitted almost isotropically. The experiments conducted to date show that the harmonics are indeed specularly reflected under certain conditions $(<150 \mathrm{fs}$, high contrast, moderate intensities $)^{17}$ and isotropically emitted for longer pulses $(\sim 1 \mathrm{ps} \text {, lower contrast, high intensity })^{14,18}$. The approximate velocity at which the critical surface deforms can be estimated from the conservation of momentum considerations ${ }^{16,19}$,

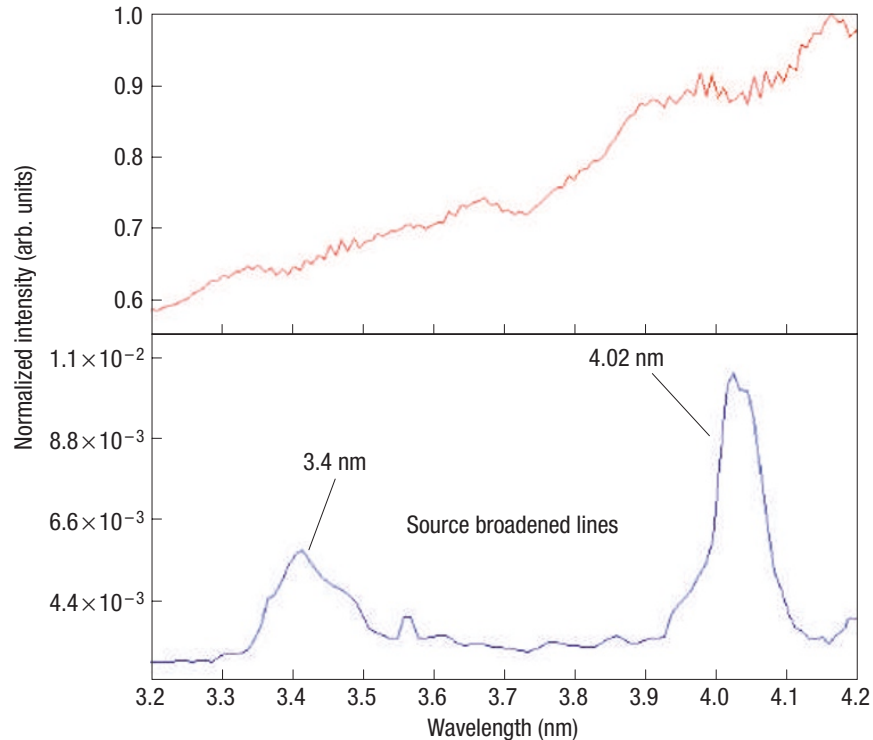

Figure 2 Quantitative comparison of spectra with and without plasma mirrors (corrected for system transmission and overlapping diffraction orders). The spectrum without PMs (bottom) is characterized by source broadened spectral lines and an approximately flat background (saturated single pixels owing to direct illumination of the CCD with hard X-rays have been removed for clarity). The spectrum taken with plasma mirrors (top) shows a much brighter ( $\sim 100$ times), harmonic quasi-continuum and no evidence of carbon emission lines. (Experimental parameters-with PMs: $E=70 \mathrm{~J}$ on a $\mathrm{CH}$ target; no PMs: $E=150 \mathrm{~J}$ on a $\mathrm{CH}$ target.)

suggesting that shorter pulses and higher effective target densities can prevent substantial surface degradation.

Finally, it can easily be understood that the harmonic production process must be phase locked, because they are the result of the reflection off a mirror surface and avoid the complications encountered with harmonic generation processes in dispersive media. Therefore, they are ideal candidates for highconversion-efficiency attosecond pulses.

To ensure a sufficiently well-defined plasma-vacuum interface, the intrinsic laser-pulse contrast ratio of $10^{7}: 1$ was enhanced to $>10^{11}: 1$ by using a double plasma mirror (PM, see Fig. 1) configuration preceding the target ${ }^{20,21}$. This may seem surprising, given the success reported in previous experiments with similar prepulse intensities on the Vulcan laser'. However, the Vulcan Petawatt laser has an intrinsic prepulse that is approximately seven times longer than that in previous publications ${ }^{14}$ and a correspondingly longer density scale length $(\sim 10 \mu \mathrm{m}$ rather than $1-2 \mu \mathrm{m}$ ) before the arrival of the main pulse and hence necessitates the use of PMs.

Consequently, strong harmonic emission in the extreme ultraviolet range is only observed in the experimental data for shots taken with PMs (Fig. 2, top), whereas much weaker line emission is visible on shots without PMs (Fig. 2, bottom). However lowerorder optical harmonics were detected on all shots. The spectrum obtained using PMs shows no evidence of carbon line emission and the peak signal level is approximately 100 times greater than that without PMs. The overall shape of the raw spectrum (Fig. 3) is dominated by the absorption features of the aluminium filter and hydrocarbon surface contaminants (which can be calculated from the depth of the carbon K-edge). Above the aluminium Ledge at $17.1 \mathrm{~nm}$ individual harmonics are clearly visible (57th to 61st). As expected, the spectrum merges into a quasi-continuum 


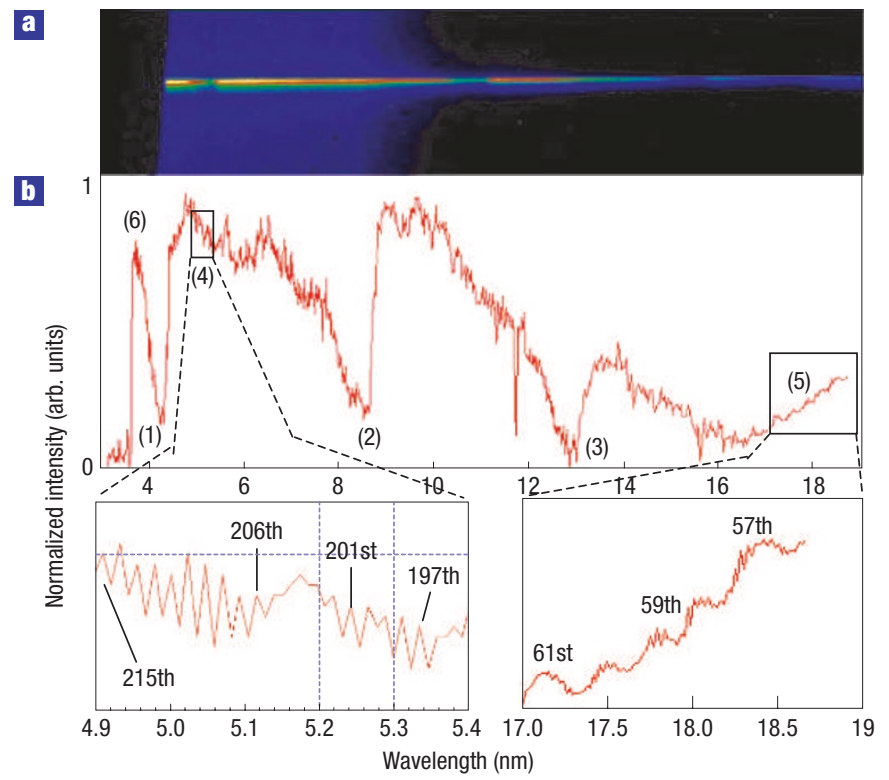

Figure 3 Unprocessed high harmonic spectrum recorded with the extreme-ultraviolet spectrometer. a, Raw CCD image obtained with the double PM setup ( $E=70 \mathrm{~J}$ on target, false colours). $\mathbf{b}$, A lineout of $\mathbf{a}$. Spectral features: (1) first-order carbon K-edge $(4.36 \mathrm{~nm})$, (2) second-order carbon K-edge, (3) third-order carbon K-edge, (4) region of resolved harmonics around 200th order (accurate to \pm 2 orders limited by calibration accuracy). The modulation depth is $>3 \sigma$ for harmonics 197-201 and 206-214, where $\sigma=N^{1 / 2}$ is the statistical pixel to pixel fluctuation expected when counting an $N$ photon/pixel signal. (5) 57th-61st harmonics (width is due to spectral defocusing which limits the spectral resolution to $\lambda / \Delta \lambda \sim 100$, spectrometer focus was optimized around $6 \mathrm{~nm}$, where $\lambda / \Delta \lambda>500$ was achieved). The modulation on top of orders $57-61$ is comparable to $\sigma$. The shadow of a block inserted to prevent direct illumination of the CCD by the zeroth order is visible at (6).

at very high harmonic orders due to the harmonic separation and width being close to the resolution limit and overlapping of diffraction orders (similar to observations of high harmonics from gaseous targets ${ }^{22}$ ). Close inspection of the spectrum reveals that it has statistically significant modulations that are spectrally closely spaced. Clear identification of individual orders is only possible in the regions of the spectrum where one order dominates the overall signal (Fig. 3b). High-contrast shots with PMs were diagnosed with the extreme-ultraviolet spectrometer in two positions (specular and on the target normal). Harmonic radiation was only observed in the specular direction, giving an upper bound for the half angle of the extreme-ultraviolet harmonic distribution of $45^{\circ}$.

The relative intensity of the harmonic orders is obtained by correcting for the spectral response of the spectrometer and by deconvolving the overlapping diffraction orders. Figure 4 shows the relative strength of the harmonics for a range of orders. The relative intensity of the harmonics shows a slope that can be well fitted by $I(n) \propto n^{-p}$, with $p=2.5(+0.2,-0.3)$. This scaling precludes other possible sources of continua, such as blackbody radiation. For example, a sufficiently bright blackbody emitter would need to be at a temperature of $3-5 \mathrm{keV}$ (depending on the precise assumptions made) and the spectrum would consequently scale as $\sim n^{+2}$ in the $50-500 \mathrm{eV}$ range.

The observed scaling demonstrates that the harmonics are generated in the relativistic limit with the expectation value for $p$ centred on the relativistic limit for quasi-monochromatic pulses.

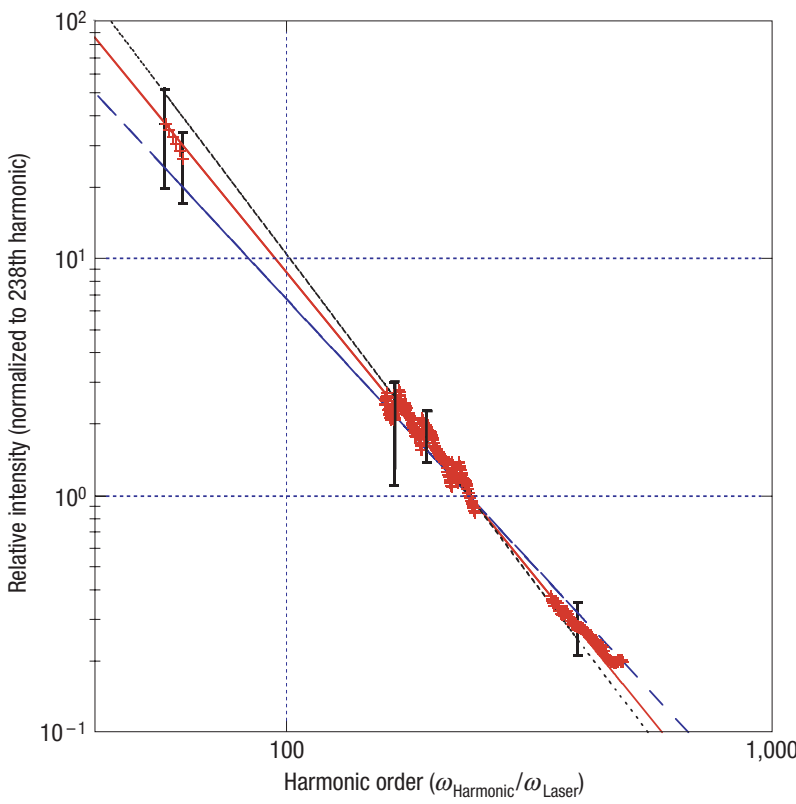

Figure 4 Relative intensity of harmonics normalized to the 238th harmonic (at the carbon $\mathrm{K}$-edge). The lines are fits to the data with the exponent $p$ as a fitting parameter such that $I(n) /\left((238)=n^{-p} / 238^{-p}\right.$. The best fit (red line) corresponds to a value of $p=2.5$ confirming harmonic production in the relativistic limit. The error bars indicate the uncertainty in the relative signal strength arising from the filter transmission and carbon contamination. The total uncertainty was calculated using standard error propagation analysis taking into account the individual uncertainty in each of the relevant quantities. The blue line corresponds to $p=2.2$ and the black line corresponds to $p=2$.7. The gaps in the spectrum correspond to spectral regions where the filters strongly absorb the harmonic radiation thus preventing a meaningful deconvolution.

The deconvolved spectrum suggests that significant contributions from wavelengths as short as $\sim 1.2 \mathrm{~nm}(\sim 850$ th harmonic $)$ are present in the spectrum in second- and third-order diffraction, whereas the shortest wavelength in first-order diffraction is $3.6 \mathrm{~nm}$. Observation of shorter wavelength harmonics is limited by the geometry and spectral response of the spectrometer. The absence of a cutoff in the spectral window investigated here is expected on the basis of recent one-dimensional calculations which suggest that up to the 10,000th harmonic can be generated at $a_{0}=20$ $\left(\sim 5 \times 10^{20} \mathrm{~W} \mathrm{~cm}^{-2} \text { with conversion efficiencies }>10^{-10}\right)^{1}$. Unlike other harmonic generation mechanisms, for example, harmonics from gaseous media ${ }^{23}$, there is no theoretical basis for a sharp cutoff in harmonic generation from solid targets. However, it has been suggested ${ }^{1}$ that the validity of the $\eta(n) \sim n^{-p}$ scaling is limited by the velocity of the oscillating surface to a maximum harmonic order of $4 \gamma^{2}$ (where $\gamma$ is the Lorentz factor), beyond which the harmonic spectrum is predicted to decay more rapidly. The ultimate extent of the harmonic spectrum remains an exciting topic for future research.

The spectral brightness of the observed harmonics can be inferred from the experimental data and varies from $10^{22}$ to $10^{24}$ photons $\mathrm{s}^{-1} \mathrm{~mm}^{-2} \mathrm{mrad}^{-2}(0.1 \%$ bandwidth) for 4 and $17 \mathrm{~nm}$ respectively (making the conservative estimate that the harmonic pulse duration and source size correspond directly to the measured laser pulse duration, $600 \mathrm{fs}$, and spot size, $8 \mu \mathrm{m}$ ). Applying the energy conversion efficiency scaling of $\eta(n) \sim n^{-p}$, it can be estimated that $\eta \sim 10^{-6 \pm 1}(0.07-0.7 \mathrm{~mJ})$ and $3 \times 10^{-5 \pm 1}(0.2-20 \mathrm{~mJ})$ 
at 4 and $17 \mathrm{~nm}$ respectively, which agrees to within experimental uncertainties with the signal observed on the detector. This scaling has been observed previously ${ }^{14}$, and is predicted to hold in the relativistic limit ${ }^{1}$. Note that these efficiencies are substantially higher than for other high-order harmonic generation mechanisms used in conjunction with short pulse lasers (for example, noble gases where efficiencies of $\sim 10^{-11}$ were reported at the carbon K-edge ${ }^{24}$ ).

In summary, high harmonic generation from solid targets in the relativistic limit has been demonstrated in good agreement with recent theoretical predictions. The slow decay of harmonic radiation with harmonic order opens up the way to exciting new physics by providing a route to high-power attosecond pulses and substantial field enhancement by means of coherent harmonic focusing. The high source brightness makes this source attractive for a range of applications that require high instantaneous brightness. However, most applications/experiments require much higher repetition rates than the 1 shot per hour provided by the Vulcan Petawatt. Proposed lasers with few-cycle pulses and a single-wavelength focal spot will only require a power of $\sim 10^{12} \mathrm{~W}$ and $\sim 10 \mathrm{~mJ}$ to achieve $10^{20} \mathrm{~W} \mathrm{~cm}^{-2} \mu \mathrm{m}^{2}$, and should be able to operate with $\mathrm{kHz}$ repetition rates ${ }^{25}$. With such lasers, specularly emitted harmonics with attosecond duration are anticipated at repetition rates and average powers suitable to testing science at attosecond timescales.

\section{METHODS}

The spectrometer consisted of a 1,200 lines $\mathrm{mm}^{-1}$ Hitachi flatfield grating ${ }^{26}$. In its original configuration, this grating is designed to produce a spectrally dispersed image in the $5-20 \mathrm{~nm}$ spectral region of a source (for example, a slit) onto a flat detector positioned $240 \mathrm{~mm}$ from the grating centre. Here the setup has been modified to image a source positioned $500 \mathrm{~mm}$ from the grating centre onto a backthinned 'ANDOR' charge-coupled device (CCD) camera. As a result, the detector plane is now tilted with regards to the original design and the focusing properties are modified ${ }^{27}$. Instead of using a slit as the source, the spectrometer was positioned to image the target. This substantially enhances the light collection efficiency and does not degrade spectral resolution for suitably small sources. The spectral resolution of the spectrometer is limited by either source size or (for sources $<20 \mu \mathrm{m}$ ) by the spatial resolution of the detector ( $27 \mu \mathrm{m}$ pixels on an ANDOR CCD camera). As the harmonic source is in the $5-10 \mu \mathrm{m}$ range, the harmonic spectra are detector limited. In this instance the spectrometer focus was optimized for the $3-10 \mathrm{~nm}$ spectral range while a loss of resolution due to defocusing is tolerated at longer wavelengths $(17-19 \mathrm{~nm})$ and clearly visible in the spectrum presented in Fig. 3.

The relative signal strengths for the harmonics shown in Fig. 4 are obtained by correcting the spectrum for the transmission function of the spectrometer (grating and gold-mirror reflectivity, $\mathrm{CH}$ (plastic) and aluminium filters ${ }^{28}, \mathrm{CCD}$ response). The diffraction efficiency of the grating was calibrated on a synchrotron for the various diffraction orders across the entire wavelength range studied here at a previous date. The filter thicknesses were measured at the target fabrication facility of the Rutherford Appleton Laboratory. The largest contribution to the uncertainty in the transmission function derives from the thickness of the hydrocarbon contamination layer on the CCD. The level of hydrocarbon contamination was estimated by fitting the shape of carbon K-edge on calibration shots. The relative intensities across the spectral range were then extracted by calculating the total number of photons incident at the carbon K-edge, where first-order diffraction dominates the signal due to the spectrometers spectral response. The signal at longer wavelengths is then calculated by subtracting the signal contribution owing to higher-order diffraction at each pixel position using the known transmission function of the spectrometer. Clearly the spectral response of the filters prevents this process for some wavelengths (for example, for the 7-17 nm spectral range where the aluminium filter has a high opacity). The systematic errors give rise to an uncertainty in the relative strength of the signal at different wavelengths and are represented as error bars in Fig. 4

Received 23 February 2006; accepted 19 May 2006; published 1 July 2006.

References

1. Gordienko, S. et al. Relativistic Doppler effect: Universal spectra and zeptosecond pulses. Phys. Rev. Lett. 93, 115002 (2004).

2. Gordienko, S. et al. Coherent focusing of high harmonics: A new way towards the extreme intensities. Phys. Rev. Lett. 94, 103903 (2005).

3. Wabnitz, H. et al. Multiple ionisation of atom clusters by intense soft X-rays from a free electron laser. Nature 420, 482-485 (2002).

4. Hentschel, M. et al. Attosecond metrology. Nature 414, 509-513 (2001)

5. Neutze, et al. Potential for biomolecular imaging with femtosecond X-ray pulses. Nature 406, 752-757 (2000).

6. Naumova, N. M. et al. Phys. Rev. Lett. 92, 063902 (2004).

7. Schwinger, J. On gauge invariance and vacuum polarization. Phys. Rev. 82, 664-679 (1951).

8. Tsakiris, G. D., Eidmann, K., Meyer-ter-Vehn, J. \& Krausz, F. Route to intense single attosecond pulses. New J. Phys. 8, 19 (2006).

9. Danson, C. N. et al. Vulcan Petawatt-an ultra-high-intensity interaction facility. Nucl. Fusion 44, S239-S242 (2004).

10. Scientific Workshop for the Extreme Light Infrastructure, ENSTA, Paris, December 9-10, 2006.

11. Gibbon, P. Harmonic generation by femtosecond laser-solid interaction: A coherent water-window light source? Phys. Rev. Lett. 76, 50-53 (1996).

12. Bulanov, S. V., Naumova, N. M. \& Pegoraro, F. Interaction of an ultrasort, relativistically intense laser-pulse with an overdense plasma. Phys. Plasmas 1, 745-757 (1994).

13. Lichters, R., Meyer-ter-Vehn, J. \& Pukhov, A. Short-pulse laser harmonics from oscillating plasma surfaces driven at relativistic intensity. Phys. Plasmas 3, 3425-3437 (1996).

14. Norreys, P. et al. Efficient extreme UV harmonics generated from picosecond laser pulse interactions with solid targets. Phys. Rev. Lett. 76, 1832-1835 (1996).

15. Zepf, M. et al. Role of the plasma scale length in the harmonic generation from solid targets. Phys. Rev. E 58, R5253-R5256 (1998).

16. Wilks, S. C. et al. Absorption of ultra intense light pulses. Phys. Rev. Lett. 69, 1383-1856 (1992)

17. Tarasevitch, A. et al. Generation of high-order spatially coherent harmonics from solid targets by femtosecond laser pulses. Phys. Rev. A 62, 023816 (2000).

18. Carman, R. L., Forslund, D. W. \& Kindel, J. M. Visible harmonics emission as a way of measuring profile steepening. Phys. Rev. Lett. 46, 29-32 (1981).

19. Zepf, M. et al. Measurements of the hole boring velocity from Doppler shifted harmonic emission from solid targets. Phys. Plasmas 3, 3242-3234 (1996).

20. Dromey, B. et al. The plasma mirror-A subpicosecond optical switch for ultrahigh power lasers. Rev. Sci. Instrum. 75, 645-648 (2004).

21. Doumy, G. et al. Complete characterization of a plasma mirror for the production of high-contrast ultraintense laser pulses. Phys. Rev. E 69, 026402 (2004).

22. Gibson, E. et al. High-order harmonic generation up to $250 \mathrm{eV}$ from highly ionized argon. Phys. Rev. Lett. 92, 033001 (2004).

23. Corkum, P. B. Plasma perspective on strong field multi-ionisation. Phys. Rev. Lett. 71, 1994-1997 (1993).

24. Spielmann, C. et al. Generation of coherent X-rays in the water window using 5 -femtosecond laser pulses. Science 278, 661-664 (1997).

25. Mourou, G. et al. On the design of experiments for the study of relativistic nonlinear optics in the limit of single-cycle pulse duration and single-wavelength spot size. Plasma Phys. Rep. 28 $12-27(2002)$

26. Kita, T. et al. Mechanically ruled aberration corrected concave gratings for a flat-field grazing incidence spectrograph. Appl. Opt. 22, 512-513 (1983).

27. Neely, D. et al. A high sensitivity imaging flat-field spectrometer design. Annual Report of the Central Laser Facilty, Rutherford Appleton Laboratory, 113-114 (1994-95).

28. Henke, B. L. et al. X-ray interactions: photoabsorption, scattering, transmission, and reflection at $\mathrm{E}=50-30000 \mathrm{eV}, \mathrm{Z}=1-92$. Atom. Data Nucl. Data Tables 54, 181-342 (1993).

\section{Acknowledgements}

We would like to acknowledge the support of the European Social Fund (B.D.). M.Z. holds a Royal Society-Wolfson Research Merit Award.

Correspondence and requests for materials should be addressed to M.Z.

Competing financial interests

The authors declare that they have no competing financial interests.

Reprints and permission information is available online at http://npg.nature.com/reprintsandpermissions/ 